CPT-97/P.3455

hep-th/9702005

January 1997

\title{
Universal correlators for multi-arc complex matrix models
}

\author{
G. Akemann 円 \\ Centre de Physique Théorique 2], CNRS, \\ Case 907 Campus de Luminy, 13288 Marseille, Cedex 9, France \\ akemann@cpt.univ-mrs.fr
}

\begin{abstract}
The correlation functions of the multi-arc complex matrix model are shown to be universal for any finite number of arcs. The universality classes are characterized by the support of the eigenvalue density and are conjectured to fall into the same classes as the ones recently found for the hermitian model. This is explicitly shown to be true for the case of two arcs, apart from the known result for one arc. The basic tool is the iterative solution of the loop equation for the complex matrix model with multiple arcs, which provides all multi-loop correlators up to an arbitrary genus. Explicit results for genus one are given for any number of arcs. The two-arc solution is investigated in detail, including the double-scaling limit. In addition universal expressions for the string susceptibility are given for both the complex and hermitian model.
\end{abstract}

\footnotetext{
${ }^{1}$ supported by European Community grant no. ERBFMBICT960997

${ }^{2}$ Unité Propre de Recherche 7061
} 


\section{Introduction}

The notion of universality is one of the crucial properties of random matrix theory. It ensures, that physical quantities of interest such as correlation functions or the free energy and its derivatives - the string susceptibility - do not depend explicitly on the matrix potential and in this sense are universal. The full dependence on the coupling constants can be encoded in few universal parameters as the endpoints of the support of the spectral density.

Universality can be found in different ways when performing the large- $N$ limit. First, in the so-called microscopic limit the oscillatory behavior of the eigenvalue density is studied and found to be universal [1] (for recent progress see [2] and references therein). In this respect particularly the study of the unitary ensembles, the complex and hermitian one-matrix model, has drawn considerable attention for its application to 3- and 4-dimensional QCD [3]. There, the merging of the eigenvalue density from two into a single arc provides an effective model for the formation of a chiral condensate in terms of random matrix theory.

In the second way of performing the large- $N$ limit, the macroscopic limit, where the oscillations of the eigenvalue density are smoothed, the splitting of the support into several pieces has been much further understood. After the seminal works for the one-arc case by Ambjørn and his collaborators for the hermitian and complex matrix model [由, 5, 6], it has been recently shown for the hermitian model, that for any number of arcs all correlation functions are universal and can be classified completely by the support of the spectral density [7, 8).

The aim of the present paper is to extend the previous results to the complex matrix model with multiple arcs, generalizing the work of [5]. The same achievements will be made here, obtaining again a whole set of universal correlators characterized by the support of the density. Moreover the new universality classes found here are conjectured to coincide precisely with the ones previously found for the hermitian model $[7,8]$. This result was already known for one arc [6] and is now proven explicitly for two arcs as well. The basic tool therefor will be again the iterative solution of the loop equation. Furthermore the string susceptibility of both, the complex and the hermitian model with two arcs will be calculated explicitly and shown to be universal.

This opens the possibility to investigate the relationship between multi-arc matrix models and integrable hierarchies using loop equation techniques [9, 10]. The outcome will have to be compared to earlier results for the two-arc solution from orthogonal polynomials [11], which depend heavily on the reliability of the ansatz for the recursion coefficients. For the case of two non-symmetric arcs this is already very doubtful [12].

Another earlier attempt towards the universality of the two-arc phase of the hermitian matrix model has been made in 13]. However, the completely independent treatment of the two arcs leads to an expression for the correlator of densities which fails to fulfill the correct analyticity properties when compared to the two-arc eigenvalue density. 
It should be mentioned that macroscopic universality has been proven for orthogonal and symplectic ensembles as well, using variational methods in the saddle-point approximation [14]. This result for the correlator of densities has been extended to planar multi-loop correlators in the framework of loop equations 15. However, the appearance of odd powers in $1 / N$ in the expansion make it very difficult to achieve explicit results for higher genera.

Furthermore renormalization group techniques for matrix models have been recently extended to the multi-arc case as well [16]. For the hermitian matrix model with a symmetric double-well potential the authors of [16] find a second attractive fixed point of the renormalization group transformation, apart from the Gaussian one. These results confirm the universality of the planar two-loop correlator for two symmetric arcs by completely different means.

The present paper is organized as follows. After briefly introducing the basic definitions in section 2 , section 3 deals with the planar solution of the loop equations for the multi-arc complex matrix model. Section 4 contains the iterative solution of the loop equation, where explicit results are given for genus one for any number of arcs. The question of the equivalence with the universality classes of the hermitian model is also addressed here. In section 5 the two-arc solution is presented in more detail including a proof for the matching with the corresponding two-arc universality class of the hermitian model. Section 6 contains all the possibilities for performing the double-scaling limit for two arcs, where the generic case maps to the double-scaled one-arc solution [6]. Finally, before concluding in the last section the string susceptibility for both the complex and the hermitian two-arc model is explicitly shown to be universal as well. Throughout the paper the notation for the solution of the one-arc complex model [5] will be followed closely.

\section{Basic Definitions}

The complex one-matrix model is defined by the partition function

$$
\begin{aligned}
Z\left[N,\left\{g_{i}\right\}\right] \equiv e^{N^{2} F} & \equiv \int d \phi^{\dagger} d \phi \exp \left(-N \operatorname{Tr} V\left(\phi^{\dagger} \phi\right)\right) \\
V\left(\phi^{\dagger} \phi\right) & \equiv \sum_{j=1}^{\infty} \frac{g_{j}}{j}\left(\phi^{\dagger} \phi\right)^{j},
\end{aligned}
$$

where the integration is over complex $N \times N$ matrices. The generating functional or one-loop correlator is given by

$$
W(p) \equiv \frac{1}{N} \sum_{k=0}^{\infty} \frac{\left\langle\operatorname{Tr}\left(\phi^{\dagger} \phi\right)^{k}\right\rangle}{p^{2 k+1}}=\frac{1}{N}\left\langle\operatorname{Tr} \frac{p}{p^{2}-\phi^{\dagger} \phi}\right\rangle .
$$

Introducing the loop insertion operator

$$
\frac{d}{d V}(p) \equiv-\sum_{j=1}^{\infty} \frac{j}{p^{2 j+1}} \frac{d}{d g_{j}}
$$


the one-loop correlator can be obtained from the free energy,

$$
W(p)=\frac{d}{d V}(p) F+\frac{1}{p}
$$

More generally one gets the $n$-loop correlator by iteratively applying the loop insertion operator to $W(p)$ (or $F$ ),

$$
\begin{aligned}
W\left(p_{1}, \ldots, p_{n}\right) & \equiv N^{n-2} \sum_{k_{1}, \ldots, k_{n}=1}^{\infty} \frac{\left\langle\operatorname{Tr}\left(\phi^{\dagger} \phi\right)^{k_{1}} \cdots \operatorname{Tr}\left(\phi^{\dagger} \phi\right)^{k_{n}}\right\rangle_{c o n n}}{p_{1}^{2 k_{1}+1} \cdots p_{n}^{2 k_{n}+1}} \\
& =\frac{d}{d V}\left(p_{n}\right) \frac{d}{d V}\left(p_{n-1}\right) \cdots \frac{d}{d V}\left(p_{2}\right) W\left(p_{1}\right), \quad n \geq 2,
\end{aligned}
$$

where conn refers to the connected part. All the multi-loop correlators and the free energy have the same $1 / N$ expansion

$$
\begin{aligned}
W\left(p_{1}, \ldots, p_{n}\right) & =\sum_{g=0}^{\infty} \frac{1}{N^{2 g}} W_{g}\left(p_{1}, \ldots, p_{n}\right), \\
F & =\sum_{g=0}^{\infty} \frac{1}{N^{2 g}} F_{g},
\end{aligned}
$$

so relation (2.6) holds for each genus separately. In particular eq. (2.4) may be written as

$$
W_{g}(p)=\frac{d}{d V}(p) F_{g}, g \geq 1
$$

\section{The Loop Equation}

The loop equation for the multi-arc complex matrix model [17] looks the same as for one arc [5] apart from the integration contour $\mathcal{C}$,

$$
\oint_{\mathcal{C}} \frac{d \omega}{4 \pi i} \frac{\omega V^{\prime}(\omega)}{p^{2}-\omega^{2}} W(\omega)=(W(p))^{2}+\frac{1}{N^{2}} \frac{d}{d V}(p) W(p), p \notin \sigma
$$

Here $\mathcal{C}$ encloses all the square root singularities (cuts) of $W(p)$ along the arcs of support $\sigma$ of the eigenvalue density $\rho(y)$,

$$
\sigma=\left\{\begin{aligned}
\bigcup_{i=1}^{\frac{s}{2}}\left(\left[-x_{2 i-1},-x_{2 i}\right] \cup\left[x_{2 i}, x_{2 i-1}\right]\right) & s \text { even } \\
\left(\bigcup_{i=1}^{\frac{s-1}{2}}\left(\left[-x_{2 i-1},-x_{2 i}\right] \cup\left[x_{2 i}, x_{2 i-1}\right]\right)\right) \cup\left[-x_{s}, x_{s}\right] & s \text { odd }
\end{aligned}\right.
$$

where $x_{1}>\ldots>x_{s}>0$. The integration contour $\mathcal{C}$ can thus be depicted in the following way 


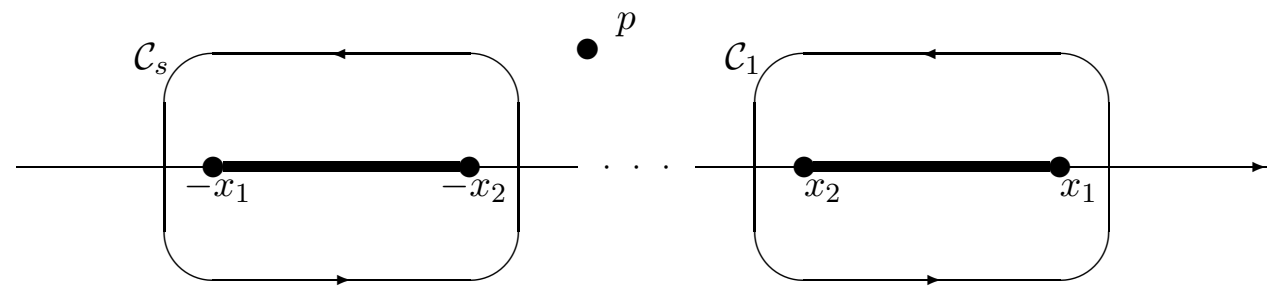

Figure 1: $\mathcal{C}=\cup_{i=1}^{s} \mathcal{C}_{i}$

As it has been explained already in [18 the eigenvalue density can be introduced either for real values $y$ or for real positive values $y^{2}, y \in \mathrm{R}$, where $y^{2}$ is the eigenvalue of $\phi^{\dagger} \phi$. In the first convention, which is chosen throughout the calculations, $\rho(y)$ is a smooth, symmetric function on $\mathrm{R}$, whereas in the latter it becomes singular when having support at the origin [18. In eq. (3.2) this is just the case when $s$ is odd. The corresponding density on $\mathrm{R}_{+}$is obtained by transforming $\rho(y) \rightarrow \rho(y) / 2 y \equiv \rho\left(y^{2}\right)$.

Now there is a simple way to see why the case of an even $s$ is actually equivalent to the hermitian model with $s / 2$ cuts on $\mathrm{R}_{+} \mathrm{I}$. When identifying

$$
\begin{aligned}
\frac{1}{p} W(p) & \left.\rightarrow W^{H}(\bar{p})\right|_{\bar{p}=p^{2}}, \\
\left( \pm x_{i}\right)^{2} & \rightarrow \bar{x}_{i}>0, i=1, \ldots, s,
\end{aligned}
$$

one exactly arrives at the loop equation of the hermitian matrix model $(H)$ with $s / 2$ cuts [8], plus an additional term $\sim 1 / \bar{p}$. This term must vanish since $W^{H}(\bar{p})$ has to be regular at $\bar{p}=0$ for $s$ even, as the origin is not included in the support $\sigma$ then'2. In [18] the same observation had been made on the level of orthogonal polynomials for the case of $s=2$, identifying the respective string equations of the complex and hermitian model. Consequently in all the following only the case of $s$ odd will be further considered. It will be called $(s+1) / 2$-arc solution, counting the number of arcs on $R_{+}$. This way of counting is chosen to name the same universality class in the hermitian and complex model by the same number of arcs.

Solving the loop equation (3.1) in the planar limit the $1 / N^{2}$-term can be omitted. The result is given by [17]

$$
W_{0}(p)=\frac{1}{2} \oint_{\mathcal{C}} \frac{d \omega}{4 \pi i} \frac{w V^{\prime}(\omega)}{p^{2}-\omega^{2}} \sqrt{\prod_{i=1}^{s} \frac{p^{2}-x_{i}^{2}}{\omega^{2}-x_{i}^{2}}},
$$

\footnotetext{
${ }^{1}$ On the level of the eigenvalue model in 18 this is intuitively clear.

${ }^{2}$ This fact had been missed in 17, giving a simple explanation why the results presented there for $s=2$ are equivalent to 6$]$.
} 
which can be rewritten as

$$
W_{0}(p)=\frac{1}{4}\left(V^{\prime}(p)-M(p) \sqrt{\prod_{i=1}^{s}\left(p^{2}-x_{i}^{2}\right)}\right) .
$$

Here $M(p)$ is an analytic function given by

$$
M(p)=\oint_{\mathcal{C}_{\infty}} \frac{d \omega}{4 \pi i} \frac{2 \omega V^{\prime}(\omega)}{\omega^{2}-p^{2}} \frac{1}{\sqrt{\prod_{i=1}^{s}\left(\omega^{2}-x_{i}^{2}\right)}} .
$$

The signs of the square root in the complex plain in eq. (3.5) are chosen to be $( \pm p)^{s}$ at $\pm \infty$. From eq. (3.5) the eigenvalue density $\rho(y)$ can be immediately read off:

$$
\begin{aligned}
\rho(y) & \equiv \lim _{\epsilon \rightarrow 0} \frac{1}{2 \pi i}\left(W_{0}(y-i \epsilon)-W_{0}(y+i \epsilon)\right) \quad, y \in \sigma \\
& =\frac{1}{4 \pi}|M(y)| \sqrt{-\prod_{i=1}^{s}\left(y^{2}-x_{i}^{2}\right)} .
\end{aligned}
$$

The parameters $x_{i}, i=1, \ldots, s$, given as functions of the coupling constants $g_{i}, i \in \mathrm{N}_{+}$, are determined by the boundary conditions on $W_{0}(p)$. From the asymptotic behavior $W(p) \sim \frac{1}{p}$ eq. (2.2) it follows

$$
\delta_{l, s}=\frac{1}{2} \oint_{\mathcal{C}} \frac{d \omega}{4 \pi i} \omega^{l} V^{\prime}(\omega) \frac{1}{\sqrt{\prod_{i=1}^{s}\left(\omega^{2}-x_{i}^{2}\right)}}, l=1,3, \ldots, s
$$

In analogy to the hermitian case [8] the remaining $\frac{s-1}{2}$ equations for the $x_{i}$ are obtained by a criterion of stability [19]

$$
0=\int_{x_{2 k+1}}^{x_{2 k}} d y M(y) \sqrt{\prod_{j=1}^{s}\left(y^{2}-x_{j}^{2}\right)}, k=1, \ldots, \frac{s-1}{2} .
$$

For $s \geq 3$ these equations lead to the explicit appearance of elliptic integrals in the higher genus correlators as well as in the planar $n$-loop correlators for $n \geq 2$.

\section{The iterative solution}

After having solved the loop equation (3.1) in leading order, an iterative scheme can be built up for calculating higher order contributions. It is based on the planar solution (3.4) along the same lines as in [5], which is treating the one-cut situation. Inserting the genus expansion of the one-loop correlator eq. (2.7) into the loop eq. (3.1) and comparing order by order in genus, the higher order contributions are determined by

$$
\left(\hat{\mathcal{K}}-2 W_{0}(p)\right) W_{g}(p)=\sum_{g \prime=1}^{g-1} W_{g^{\prime}}(p) W_{g-g^{\prime}}(p)+\frac{d}{d V}(p) W_{g-1}(p), g \geq 1 .
$$

\footnotetext{
${ }^{3}$ Because of symmetry the stability criterion has to be imposed only between half of all arcs.
} 
Here $\hat{\mathcal{K}}$ is a linear integral operator, given by

$$
\hat{\mathcal{K}} f(p) \equiv \oint_{\mathcal{C}} \frac{d \omega}{4 \pi i} \frac{\omega V^{\prime}(\omega)}{p^{2}-\omega^{2}} f(\omega) .
$$

Once the result for $W_{0}(p)$ is known one can determine $W_{g}(p)$ for $g \geq 1$ iteratively from contributions of lower genus on the r.h.s. of eq. (4.1), provided that the operator $\left(\hat{\mathcal{K}}-2 W_{0}(p)\right)$ can be uniquely inverted. However, it can be shown from the boundary conditions (3.8), that this operator possesses the following zero modes

$$
\begin{aligned}
\operatorname{Ker}\left(\hat{\mathcal{K}}-2 W_{0}(p)\right) & =\operatorname{Span}\left\{\phi^{(0)}(p), p^{2} \phi^{(0)}(p), \ldots, p^{s-1} \phi^{(0)}(p)\right\} \\
\phi^{(0)}(p) & \equiv \frac{1}{\sqrt{\prod_{i=1}^{s}\left(p^{2}-x_{i}^{2}\right)}} .
\end{aligned}
$$

Because of the asymptotic of $W_{g}(p) \sim \mathcal{O}\left(\frac{1}{p^{3}}\right)$ for $g \geq 1$, which can be seen from the definition (2.2), the last zero mode $\sim \frac{1}{p}$ cannot contribute to $W_{g}(p)$. In the case of $s=1$ this argument uniquely fixes the inversion of eq. (4.1) [5]. Here, the condition (2.9) will be used in addition to achieve a unique solution $W_{g}(p)$ for any $s$. Therefore a set of basis functions is introduced in the following way:

$$
\begin{aligned}
& \left(\hat{\mathcal{K}}-2 W_{0}(p)\right) \chi_{i}^{(n)}(p)=\frac{1}{\left(p^{2}-x_{i}^{2}\right)^{n}}, i=1, \ldots, s, n \in \mathrm{N}_{+}, \\
& \left(\hat{\mathcal{K}}-2 W_{0}(p)\right) \psi^{(n)}(p)=\frac{1}{p^{2 n}} \quad, n \in \mathrm{N}_{+} .
\end{aligned}
$$

In addition a change of variables from the coupling constants $g_{i}$ to the following moments is performed:

$$
\begin{aligned}
I_{i}^{(k)} & \equiv \oint_{\mathcal{C}} \frac{d \omega}{4 \pi i} \frac{\omega V^{\prime}(\omega)}{\left(\omega^{2}-x_{i}^{2}\right)^{k}} \phi^{(0)}(\omega), \quad i=1, \ldots, s, k \in \mathrm{N}_{+}, \\
M^{(n)} & \equiv \oint_{\mathcal{C}} \frac{d \omega}{4 \pi i} \frac{V^{\prime}(\omega)}{\omega^{2 n+1}} \phi^{(0)}(\omega) \quad, \quad n \in \mathrm{N} .
\end{aligned}
$$

Apart from the endpoints of the cuts $x_{i}$ these moments will encode the universality of the higher genus correlators. Namely given that the r.h.s. of eq. (4.1) is a fractional rational function of $p$ having poles at the $x_{i}$ only, $W_{g}(p)$ will have the following structure:

$$
W_{g}(p)=\sum_{n=1}^{3 g-1} \sum_{i=1}^{s} A_{i, g}^{(n)} \chi_{i}^{(n)}(p)+\sum_{n=1}^{g} D_{g}^{(n)} \psi^{(n)}(p) .
$$

The coefficients of the basis functions $A_{i, g}^{(n)}$ and $D_{g}^{(n)}$ are complicated functions of the endpoints $x_{i}$ and of the moments eq. (4.5). As the order of the highest pole in $W_{g}(p)$ is not changed compared to the one-cut case [5], $W_{g}(p)$ will depend on at most $s(3 g-1)$ moments $I_{i}^{(k)}$ and $g$ moments $M^{(n)}$. This follows from the same arguments as in [5]. The unique set of basis functions can be 
proven to be (see appendix A):

$$
\begin{aligned}
\chi_{i}^{(n)}(p) \equiv & \frac{1}{I_{i}^{(1)}}\left(\left.\phi_{i}^{(n)}(p)\right|_{\frac{d}{d V}-p a r t}-\sum_{k=1}^{n-1} \chi_{i}^{(k)}(p) I_{i}^{(n-k+1)}\right), \\
\psi^{(n)}(p) \equiv & \frac{1}{M^{(0)}}\left(\left.\Omega^{(n)}(p)\right|_{\frac{d}{d V}-p a r t}-\sum_{k=1}^{n-1} \psi^{(k)}(p) M^{(n-k)}\right), \\
& \phi_{i}^{(n)}(p) \equiv \frac{1}{\left(p^{2}-x_{i}^{2}\right)^{n}} \phi^{(0)}(p), \quad i=1, \ldots, s, n \in \mathrm{N}_{+}, \\
& \Omega^{(n)}(p) \equiv \frac{1}{p^{2 n}} \phi^{(0)}(p), \quad n \in \mathrm{N}_{+} .
\end{aligned}
$$

The restriction to the part which can be written as a derivative of the loop insertion operator $\frac{d}{d V}(p)$ ensures that the whole $p$-dependence of the basis functions and thus of $W_{g}(p)$ in eq. (4.6) can be absorbed into such derivatives in order to fulfill eq. (2.9). At the same time this requirement uniquely fixes the ambiguities coming from the zero modes in eq. (4.3).

The explicit construction of the basis functions can be found in appendix A, the first of which reading

$$
\begin{aligned}
\chi_{i}^{(1)}(p) & =\frac{1}{x_{i}^{2}} \frac{d x_{i}^{2}}{d V}(p), \quad i=1, \ldots, 2 s \\
\chi_{i}^{(2)}(p) & =\frac{-2}{3 x_{i}^{2} I_{i}^{(1)}} \frac{d I_{i}^{(1)}}{d V}(p)-\frac{2}{3 x_{i}^{4}} \frac{d x_{i}^{2}}{d V}(p)-\frac{1}{3 x_{i}^{2}} \sum_{\substack{j=1 \\
j \neq i}}^{s} \frac{1}{x_{j}^{2}-x_{i}^{2}}\left(\frac{d x_{j}^{2}}{d V}(p)-\frac{d x_{i}^{2}}{d V}(p)\right) \\
\psi^{(1)}(p) & =-\frac{2}{M^{(0)}} \frac{d M^{(0)}}{d V}(p)-\sum_{i=1}^{s} \frac{1}{x_{i}^{2}} \frac{d x_{i}^{2}}{d V}(p)
\end{aligned}
$$

The one-loop correlator of genus one $W_{1}(p)$ can now be calculated from eq. (4.1) for $g=1$, which reads

$$
\left(\hat{\mathcal{K}}-2 W_{0}(p)\right) W_{1}(p)=\frac{d}{d V}(p) W_{0}(p) .
$$

Therefore the planar two-loop correlator $W_{0}(p, p)$ on the r.h.s. has to be determined, which can be achieved from applying $\frac{d}{d V}(p)$ to eq. (3.4) in the following form

$$
\begin{aligned}
& \frac{d}{d V}(p)= \frac{\partial}{\partial V}(p)+\sum_{i=1}^{s}\left(\frac{d x_{i}^{2}}{d V}(p) \frac{\partial}{\partial x_{i}^{2}}+\sum_{k=1}^{\infty} \frac{d I_{i}^{(k)}}{d V}(p) \frac{\partial}{\partial I_{i}^{(k)}}\right)+\sum_{k=0}^{\infty} \frac{d M^{(k)}}{d V}(p) \frac{\partial}{\partial M^{(k)}} \\
& \frac{\partial}{\partial V}(p) \equiv-\sum_{j=1}^{\infty} \frac{j}{p^{j+1}} \frac{\partial}{\partial g_{j}} .
\end{aligned}
$$

Using the results from the appendix A eq. (A.7) one obtains

$$
\begin{aligned}
W_{0}(p, p)= & \frac{1}{16} \sum_{i=1}^{s} \frac{x_{i}^{2}}{\left(p^{2}-x_{i}^{2}\right)^{2}}-\frac{1}{16} \sum_{\substack{i, j=1 \\
i \neq j}}^{s} \frac{x_{i}^{2}}{x_{i}^{2}-x_{j}^{2}}\left(\frac{1}{p^{2}-x_{i}^{2}}-\frac{1}{p^{2}-x_{j}^{2}}\right) \\
& +\frac{1}{16 p^{2}}-\frac{s}{16} \sum_{i=1}^{s} \frac{1}{p^{2}-x_{i}^{2}}+\frac{1}{4} \sum_{i=1}^{s} \sum_{l=0}^{\frac{s-3}{2}} \frac{\alpha_{i, l} x_{i}^{2 l}}{p^{2}-x_{i}^{2}}
\end{aligned}
$$


where the $\alpha_{i, l}$ are given by the solution of the linear set of equations (A.8). They depend only on the endpoints of the cuts $x_{i}$ and on elliptic functions of them. The result for $W_{1}(p)$ now follows from inverting eq. (4.9) by inserting the basis functions eq. (4.8) into $W_{0}(p, p)$ :

$$
\begin{aligned}
W_{1}(p) & =\frac{1}{16} \sum_{i=1}^{s} x_{i}^{2} \chi_{i}^{(2)}(p)-\frac{1}{16} \sum_{\substack{i, j=1 \\
i \neq j}}^{s} \frac{x_{i}^{2}}{x_{i}^{2}-x_{j}^{2}}\left(\chi_{i}^{(1)}(p)-\chi_{j}^{(1)}(p)\right) \\
& +\frac{1}{16} \psi^{(1)}(p)-\frac{s}{16} \sum_{i=1}^{s} \chi_{i}^{(1)}(p)+\frac{1}{4} \sum_{i=1}^{s} \sum_{l=0}^{\frac{s-3}{2}} \alpha_{i, l} x_{i}^{2 l} \chi_{i}^{(1)}(p) .
\end{aligned}
$$

The calculation of higher genera $W_{g}(p)$ as well as the explicit integration to get the free energy $F_{g}$ becomes very technical as one has to differentiate or to integrate the set of elliptic functions given in eq. (A.6). Still, following the arguments from [5] it becomes clear, that the general structure for $W_{g}(p)$ will be precisely the one given in eq. (4.6) for an arbitrary number of cuts.

In the case $s=3$ the elliptic integrals occurring are given in terms of the well known complete elliptic integrals of first, second and third kind (see eq. (B.2)) making more explicit results accessible. In the generic double-scaling limit the solution will be mapped to the double-scaled one-cut solution [6]. This relation is probably true for all multi-cut solutions. In [6] higher genus results have been obtained explicitly up to and including $g=4$.

Apart from the general result for genus one for any $s$ also the planar twoloop correlator at different arguments can be calculated explicitly, using again the results from appendix A. Performing a similar calculation as for $W_{0}(p, p)$ it follows

$$
\begin{gathered}
W_{0}(q, p)=\frac{1}{4} \frac{\phi^{(0)}(q)}{\phi^{(0)}(p)}\left(\frac{2 q^{2}}{\left(q^{2}-p^{2}\right)^{2}}+\frac{1}{q^{2}-p^{2}}\left(s-1+\sum_{i=1}^{s} \frac{x_{i}^{2}}{p^{2}-x_{i}^{2}}\right)\right. \\
\left.+\sum_{i=1}^{s} \sum_{l=0}^{\frac{s-3}{2}} \frac{\alpha_{i, l} q^{2 l}}{p^{2}-x_{i}^{2}}\right)-\frac{1}{2} \frac{p q}{\left(q^{2}-p^{2}\right)^{2}} .
\end{gathered}
$$

Thus $W_{0}(p, q)$ and because of eqs. (2.6) and (4.10) also all the higher planar multi-loop correlators are universal in the sense that they only depend on the endpoints of the cuts $x_{i}$. The same statement can be made for the density correlators, as they can be obtained from the multi-loop correlators and vice versa (see e.g. [7]). Consequently eq. (4.13) gives a classification of all universality classes of the multi-arc complex matrix model in terms of the support of the eigenvalue density.

A similar classification has been found in previous works for the hermitian model [7, 8], so a natural question to ask is whether one can identify the classes here and there, which have the same number of intervals as support. In order to do so one has to map again the correlation functions from the hermitian model [8] to the corresponding quantities in the complex model as in eq. (3.3),

$$
\left.\frac{1}{p^{2}} W_{0}(p, p) \rightarrow W_{0}^{H}(\bar{p}, \bar{p})\right|_{\bar{p}=p^{2}} .
$$


In addition the smallest endpoint $\bar{x}_{2 \bar{s}}$ of the cuts of the hermitian model $(H)$ has to be set to zero, as now the complex model with odd $s$ is treated . While for the one-cut case it is known already, that the universality classes for the planar two-loop correlators match [4], in the general case $W_{0}(p, p)$ from eq. (4.11) has to be compared with 8

$$
\begin{aligned}
\bar{p} W_{0}^{H}(\bar{p}, \bar{p}) & =\frac{1}{16} \sum_{i=1}^{2 \bar{s}-1} \frac{\bar{x}_{i}}{\left(\bar{p}-\bar{x}_{i}\right)^{2}}-\frac{1}{16} \sum_{\substack{i, j=1 \\
i \neq j}}^{2 \bar{s}-1} \frac{\bar{x}_{i}}{\bar{x}_{i}-\bar{x}_{j}}\left(\frac{1}{\bar{p}-\bar{x}_{i}}-\frac{1}{\bar{p}-\bar{x}_{j}}\right) \\
& +\frac{1}{16 \bar{p}}-\frac{2 \bar{s}-1}{16} \sum_{i=1}^{2 \bar{s}-1} \frac{1}{\bar{p}-\bar{x}_{i}}+\frac{1}{4} \sum_{i=1}^{2 \bar{s}-1} \sum_{l=0}^{\frac{2 \bar{s}-4}{2}} \frac{\bar{\alpha}_{i, l} \bar{x}_{i}^{l}}{\bar{p}-\bar{x}_{i}}+\frac{\alpha_{2 \bar{s}, 0}}{4 \bar{p}}(4.15)
\end{aligned}
$$

for $\bar{p}=p^{2}, \bar{x}_{i}=x_{i}^{2}, i=1, \ldots, 2 \bar{s}-1$ and $\bar{x}_{2 \bar{s}} \rightarrow 0$. When identifying $s=2 \bar{s}-1$, which is the corresponding number of endpoints, the two correlators eq. (4.11) and eq. (4.15) are identical apart from the last extra term in eq. (4.15) which is non vanishing in the limit $x_{2 \bar{s}} \rightarrow 0$. However, one has to bare in mind that the $\bar{\alpha}_{i, l}$ are solutions to a set of $2 \bar{s}(\bar{s}-1)$ linear equations (see eq. (5.16) in [8]), which are different from the $s(s-1) / 2$ eqs. (A.8) determining the $\alpha_{i, l}$. A proof for the equivalence of the two sets of universality classes would thus need some nontrivial identities for the elliptic integrals occurring and is therefore left as a conjecture. Still, for the case of $s=3$ this proof can performed explicitly as it will be shown in the next section. Summing up the results obtained so far the complex model with both $s$ even and odd contains all universality classes found for the unitary ensembles, due to the fact that the hermitian model is contained in the complex one for the case of $s$ even.

\section{The two-cut solution $s=3$}

As it has been mentioned already for $s=3$ further results become accessible as the elliptic integrals originating from the boundary conditions can be related to the complete elliptic integrals of the first, second and third kind. Explicit expressions for the zero mode coefficients $\alpha_{i}$ of the quantities $\frac{d x_{i}^{2}}{d V}(p)$, which are responsible for the appearance of the elliptic integrals, are given in appendix B eq. (B.5).

In the previous section the set of universality classes for all multi-arc solutions for the complex model has been given and an equivalence to the ones recently found in the hermitian model [7, 8] was conjectured. In the special case considered this equivalence will be shown to hold. For $s=3$ the general results obtained for $W_{0}(p, p)$ eq. (4.11) and $W_{0}(p, q)$ eq. (4.13) take the following form, using the results for the $\alpha_{i}$ from appendix B,

\footnotetext{
${ }^{4}$ In matching the correlators of the two models the density for nonnegative eigenvalues of the complex model is considered.
} 


$$
\begin{aligned}
W_{0}(p, p)= & \frac{1}{16} \sum_{i=1}^{3} \frac{x_{i}^{2}}{\left(p^{2}-x_{i}^{2}\right)^{2}}+\frac{1}{16 p^{2}}-\frac{1}{16} \sum_{i<j}^{3} \frac{x_{i}^{2}+x_{j}^{2}}{\left(p^{2}-x_{i}^{2}\right)\left(p^{2}-x_{j}^{2}\right)} \\
& +\frac{1}{16} \frac{1}{p^{2}-x_{1}^{2}}+\frac{3\left(x_{2}^{2}+x_{3}^{2}\right)-2 p^{2}}{16\left(p^{2}-x_{2}^{2}\right)\left(p^{2}-x_{3}^{2}\right)}+\frac{1}{4} \frac{x_{2}^{2}\left(x_{1}^{2}-x_{3}^{2}\right)}{\prod_{j=1}^{3}\left(p^{2}-x_{j}^{2}\right)} \frac{E(k)}{K(k)} \\
W_{0}(p, q)= & \frac{1}{4\left(p^{2}-q^{2}\right)^{2}}\left(p^{2} \sqrt{\frac{\left(p^{2}-x_{1}^{2}\right)\left(q^{2}-x_{2}^{2}\right)\left(q^{2}-x_{3}^{2}\right)}{\left(p^{2}-x_{2}^{2}\right)\left(p^{2}-x_{3}^{2}\right)\left(q^{2}-x_{1}^{2}\right)}}\right. \\
& \left.+q^{2} \sqrt{\frac{\left(p^{2}-x_{2}^{2}\right)\left(p^{2}-x_{3}^{2}\right)\left(q^{2}-x_{1}^{2}\right)}{\left(p^{2}-x_{1}^{2}\right)\left(q^{2}-x_{2}^{2}\right)\left(q^{2}-x_{3}^{2}\right)}}\right) \\
+ & \frac{1}{4} \frac{x_{2}^{2}\left(x_{1}^{2}-x_{3}^{2}\right)}{\sqrt{\prod_{j=1}^{3}\left(p^{2}-x_{j}^{2}\right)\left(q^{2}-x_{j}^{2}\right)}} \frac{E(k)}{K(k)}-\frac{1}{2} \frac{p q}{\left(p^{2}-q^{2}\right)^{2}}
\end{aligned}
$$

The consistency of the two results can be checked explicitly. Namely $W_{0}(p, q)$ is symmetric, it has the same analyticity properties as $W_{0}(p)$, it asymptotically reaches $\sim \mathcal{O}\left(\frac{1}{p^{3}}\right)$, it is regular at equal arguments and coincides with $W_{0}(p, p)$ and it finally has the correct discontinuity $-\frac{p q}{\left(p^{2}-q^{2}\right)^{2}}$ along the cuts. The conjectured equivalence of the universality class of the hermitian model with two cuts at $\left[\bar{x}_{4}, \bar{x}_{3}\right] \cup\left[\bar{x}_{2}, \bar{x}_{1}\right]$ is now evident when comparing with the results given in 8] (eqs. (6.6) and (6.5)). Setting $\bar{x}_{4}=0$ and identifying $\bar{x}_{i}=x_{i}^{2}$ it holds

$$
\begin{aligned}
\frac{1}{p^{2}} W_{0}(p, p) & \left.\equiv W_{0}^{H}(\bar{p}, \bar{p})\right|_{\substack{\bar{p}=p^{2} \\
\bar{x}_{i}=x_{i}^{2}, \bar{x}_{4}=0}}, \quad, \\
\frac{1}{p q} W_{0}(p, q) & \left.\equiv W_{0}^{H}(\bar{p}, \bar{q})\right|_{\substack{\bar{p}=p^{2}, \bar{q}=q^{2} \\
\bar{x}_{i}=x_{i}^{2}, \bar{x}_{4}=0}}
\end{aligned} .
$$

So despite the fact that in the complex model the eigenvalue density $\rho\left(y^{2}\right)$ becomes singular at the origin whereas the density of the hermitian model stays smooth, both models belong to the same universality class for one and two cuts. Nevertheless, for higher genera the two models will give in general different results, as it can be seen for example from comparing the free energy obtained below with the one in [8]. It is only in the double-scaling limit, that all the models mentioned will become completely equivalent (see sect. 6).

The correlator of densities given in [13], which can be related to the planar two-loop correlator $W_{0}(p, q)$ (see e.g. [7]), does not match with the above twoarc universality class for the following reasons. First of all the authors of 13 do not impose any boundary condition as in eq. (3.9) and are therefore left with a parameter dependent expression. Furthermore their independent treatment of the two arcs, adding up two single arc correlators with different endpoints, does not fulfill the correct analyticity properties compared to the two-arc eigenvalue density in $[8]$.

Turning back to the results for genus $g=1$ from the previous section also the free energy $F_{1}$ can now be determined. This is due to the fact that the 
derivatives of the complete elliptic integrals with respect to their moduli are known and close among themselves (see e.g. [20]). From eq. (4.12) the oneloop correlator of genus one for $s=3$ takes the following form,

$$
\begin{aligned}
W_{1}(p)= & -\frac{1}{24} \sum_{i=1}^{3}\left(\frac{1}{I_{i}^{(1)}} \frac{d I_{i}^{(1)}}{d V}(p)+\frac{1}{x_{i}^{2}} \frac{d x_{i}^{2}}{d V}(p)+\sum_{j>i} \frac{1}{x_{j}^{2}-x_{i}^{2}}\left(\frac{d x_{j}^{2}}{d V}(p)-\frac{d x_{i}^{2}}{d V}(p)\right)\right) \\
& -\frac{1}{16} \sum_{i \neq j} \frac{1}{x_{i}^{2}-x_{j}^{2}}\left(\frac{d x_{i}^{2}}{d V}(p)-\frac{x_{i}^{2}}{x_{j}^{2}} \frac{d x_{j}^{2}}{d V}(p)\right)-\frac{1}{8} \frac{1}{M^{(0)}} \frac{d M^{(0)}}{d V}(p) \\
& -\frac{1}{4} \sum_{i=1}^{3} \frac{1}{x_{i}^{2}} \frac{d x_{i}^{2}}{d V}(p)+\frac{1}{4} \sum_{i=1}^{3} \frac{\alpha_{i}}{x_{i}^{2}} \frac{d x_{i}^{2}}{d V}(p)
\end{aligned}
$$

Using the results for the $\alpha_{i}$ eq. (B.5) and standard formula for the complete elliptic integrals [20] $F_{1}$ can be integrated according to eq. (2.9)

$$
\begin{aligned}
F_{1}= & -\frac{1}{24} \sum_{i=1}^{3} \ln \left|I_{i}^{(1)}\right|-\frac{1}{8} \ln \left|M^{(0)}\right|-\frac{1}{2} \ln |K(k)|-\frac{1}{6} \sum_{i=1}^{3} \ln \left|x_{i}^{2}\right| \\
& -\frac{1}{6} \sum_{i<j} \ln \left|x_{i}^{2}-x_{j}^{2}\right|+\frac{1}{4}\left(\ln \left|x_{1}^{2}-x_{3}^{2}\right|+\ln \left|x_{2}^{2}\right|\right) .
\end{aligned}
$$

Apart from the factor of the $M^{(0)}$-term all the other terms coincide with the free energy of genus one of the hermitian model [8] at $\bar{x}_{4}=0$. The same observation can be made when comparing the free energies of the one-cut solutions. There, at higher genera the difference between the hermitian and complex model becomes more evident. The iterative procedure of solving the loop equation is purely algebraic and in the case of $s=3$ even accessible to computer algebra, grace to the knowledge of the elliptic integrals. In the next section higher genera will be obtained by other means, namely by comparing to the results for the one-cut solution in the double-scaling limit [6].

\section{The double-scaling limit for $s=3$}

In general two different situations may occur in the double-scaling limit (d.s.l.). In the generic case, when the limit is performed at any of the endpoints $\pm x_{i}$, the contributions coming from the multi-cut structure will drop out and the correlators will precisely match with the continuum version of the onecut case [6]. A different situation is given, when the d.s.l. is performed at simultaneously shrinking or/and merging arcs. The correlators will differ from the one-arc result, as it has been found similarly in the d.s.l. of the hermitian two-cut model [8].

\subsection{The scaling limit at $x_{j}$}

Choosing any endpoint, say $x_{j}$, the $m$-th multi-critical point is defined by $m-1$ extra zeros of the eigenvalue density eq. (3.7) accumulating at $+x_{j}$ and $-x_{j}$. 
By tuning the coupling constants in order to reach this point the argument of the correlators $p$ and the $\pm x_{j}$ will scale like

$$
\begin{aligned}
& p^{2}=\left(x_{j}^{c}\right)^{2}+a \pi, \\
& x_{j}^{2}=\left(x_{j}^{c}\right)^{2}-a \Lambda^{\frac{1}{m}},
\end{aligned}
$$

with $a \rightarrow 0$, whereas the $x_{i \neq j}^{2}$ do not scale. Since $I_{i}^{(k)}=\left.\frac{1}{2} \frac{1}{(k-1) !} \frac{d^{k-1} M(p)}{d p^{2(k-1)}}\right|_{p=x_{i}}$ the $j$-th moments will scale like

$$
I_{j}^{(k)} \sim a^{m-k}
$$

whereas the other moments $I_{i \neq j}^{(k)}$ and $M^{(n)}$ do not scale either. In the limit eq. 6.1) the elliptic integrals $K(k)$ and $E(k)$ and consequently the zero mode contributions $\alpha_{i}$ will stay subdominant in eqs. (A.7), (A.3) and (A.4),

$$
\begin{aligned}
\frac{d x_{j}^{2}}{d V}(p) & =\frac{x_{j}^{2}}{I_{j}^{(1)}} \phi_{j}^{(1)}(p) \quad(\text { d.s.l. }) \\
\frac{d I_{j}^{(k)}}{d V}(p) & =\left(k+\frac{1}{2}\right)\left(I_{j}^{(k+1)} \frac{d x_{j}^{2}}{d V}(p)-x_{j}^{2} \phi_{j}^{(k+1)}(p)\right) \quad \text { (d.s.l.) } .
\end{aligned}
$$

Only the basis functions $\chi_{j}^{(k)}(p) \sim a^{-m-n+\frac{1}{2}}$ will give dominant contributions when inverting the loop equation. Moreover the loop insertion operator itself will simplify due to eq. (6.4) as well as $W_{0}(p, p)$ in eq. (4.11), the starting point of the iteration,

$$
\begin{aligned}
W_{0}(p, p) & =\frac{1}{16} \frac{x_{j}^{2}}{\left(p^{2}-x_{j}^{2}\right)^{2}} \quad \text { (d.s.l.) } \\
\frac{d}{d V}(p) & =\frac{d x_{j}^{2}}{d V}(p) \frac{\partial}{\partial x_{j}^{2}}+\sum_{k=1}^{\infty} \frac{d I_{j}^{(k)}}{d V}(p) \frac{\partial}{\partial I_{j}^{(k)}} \quad \text { (d.s.l.) } .
\end{aligned}
$$

The eqs. (6.3) - 6.5) precisely map to the corresponding quantities of the double-scaled one-cut solution, which are given in the appendix A of [6]. Consequently both solutions are equivalent in any order of genus, as they start the iteration from the same quantities. In fact more was proven in [6], namely that in the d.s.l. the complex and the hermitian matrix model become completely equivalent. From the above results this equivalence now extends to the complex model for $s=3$. The same matching has been shown for the two-cut hermitian model [8]. This equivalence has to be understood in the symbolical sense that the basic objects as moments and endpoints of support appear exactly in the same way. However, when expanding these objects in terms of coupling constants $g_{i}$, the expansion explicitly depends on the number of cuts.

\subsection{The scaling limit at merging and shrinking arcs}

In the case where the d.s.l. is considered at an endpoint of an arc, which is simultaneously shrinking to zero, merging with another arc, or both together, the following situations are possible: 

i) $x_{2} \rightarrow x_{3}$ merging
ii) $x_{3} \rightarrow 0$ shrinking
iii) $x_{2} \rightarrow x_{1}$ shrinking
iv) $x_{2}, x_{3} \rightarrow 0$ merging + shrinking
v) $x_{1}, x_{2} \rightarrow x_{3}$ merging + shrinking

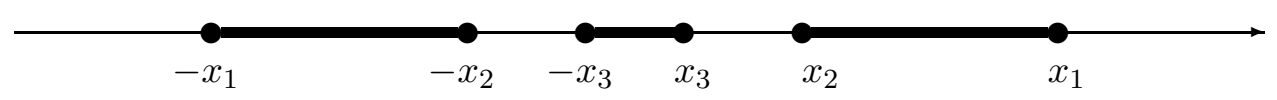

Figure 2: The support $\sigma$ of the eigenvalue density $\rho(y)$ for $s=3$

In all five cases the resulting continuum limit will differ from the previous one called generic, and thus from the d.s.l. of the one-cut solution. Only in the last case v) the contributions containing elliptic integrals in eqs. A.7), (A.3) and (A.4) will stay dominant. In all five cases the iterative solution of the loop equation with not simplify much any longer. The case i) will be treated in more detail as an example, whereas in the remaining cases only the scaling behavior of the zero mode coefficients $\alpha_{i}$ of the quantities $\frac{d x_{i}^{2}}{d V}(p)$ will be given.

i) The transition of merging cuts can be parameterized by

$$
\begin{aligned}
& x_{2}^{2}=x_{c}^{2}+a \nu, \\
& x_{3}^{2}=x_{c}^{2}-a \mu, \quad \nu, \mu>0, \\
& p^{2}=x_{c}^{2}+a \pi .
\end{aligned}
$$

In this limit the modulus $k$ of the elliptic integrals vanishes,

$$
k^{2}=a(\nu+\mu) \frac{x_{1}^{2}}{x_{c}^{2}\left(x_{1}^{2}-x_{c}^{2}\right)}+\mathcal{O}\left(a^{2}\right),
$$

and consequently $K(k)$ and $E(k)$ can be expanded in terms of $k^{2}$,

$$
\frac{E(k)}{K(k)}=1-\frac{1}{2} k^{2}+\mathcal{O}\left(a^{2}\right) .
$$

The coefficients $\alpha_{i}$ will stay finite in the limit (6.6),

$$
\begin{aligned}
\alpha_{1} & =\frac{x_{1}^{2}}{x_{1}^{2}-x_{c}^{2}}+\mathcal{O}(a) \\
\alpha_{2} & =\frac{1}{2}-\frac{x_{c}^{2}}{2\left(x_{1}^{2}-x_{c}^{2}\right)}+\mathcal{O}(a), \\
\alpha_{3} & =\alpha_{2}+\mathcal{O}(a),
\end{aligned}
$$


leaving the zero mode contributions $\alpha_{i} \phi^{(0)}(p) \sim a^{-1}$ subdominant compared to $\phi_{j=2,3}^{(1)} \sim a^{-2}$ in eq. (B.3). Due to the scaling of both sets of moments $I_{j=2,3}^{(k)}$ their derivative now reads

$$
\begin{aligned}
\frac{d I_{2}^{(k)}}{d V}(p)= & \left(k+\frac{1}{2}\right)\left(I_{2}^{(k+1)} \frac{d x_{2}^{2}}{d V}(p)-x_{2}^{2} \phi_{2}^{(k+1)}(p)\right) \\
& +\frac{1}{2} \sum_{l=1}^{k} \frac{1}{\left(x_{3}^{2}-x_{2}^{2}\right)^{k-l+1}}\left(x_{3}^{2} \phi_{2}^{(l)}(p)-I_{2}^{(l)} \frac{d x_{3}^{2}}{d V}(p)\right) \text { (d.s.l.) },
\end{aligned}
$$

and the same for the indices 2 and 3 interchanged. The starting point for the iteration $W_{0}(p, p)$ becomes

$$
W_{0}(p, p)=\frac{1}{16} \sum_{i=2,3} \frac{x_{i}^{2}}{\left(p^{2}-x_{i}^{2}\right)}-\frac{1}{16} \frac{\left(x_{2}^{2}+x_{3}^{2}\right)}{\left(x_{2}^{2}-x_{3}^{2}\right)}\left(\frac{1}{p^{2}-x_{2}^{2}}-\frac{1}{p^{2}-x_{3}^{2}}\right)
$$

from which $W_{1}(p)$ can be easily obtained. Clearly the result differs from the generic d.s.l. in the previous subsection.

ii) The d.s.l. at the inner arc shrinking to zero can be parameterized as

$$
\begin{aligned}
& x_{3}^{2}=a, \\
& p^{2}=a \pi .
\end{aligned}
$$

As the modulus $k^{2}$ reaches unity in this limit the elliptic integrals can be expanded in terms of the complementary modulus $k^{2}$

$$
\begin{aligned}
k^{\prime 2} & \equiv 1-k^{2}=a \frac{x_{1}^{2}-x_{2}^{2}}{x_{1}^{2} x_{2}^{2}} \\
\Rightarrow \frac{E(k)}{K(k)} & \sim \mathcal{O}\left(\frac{1}{\ln a}\right) .
\end{aligned}
$$

The $\alpha_{i}$ will stay finite or vanish,

$$
\begin{aligned}
& \alpha_{1}=\alpha_{2}=1+\mathcal{O}\left(\frac{1}{\ln a}\right), \\
& \alpha_{3} \sim \mathcal{O}\left(\frac{1}{\ln a}\right) .
\end{aligned}
$$

Still, the continuum limit will be changed compared to the generic d.s.l. as now the moments $M^{(k)}$ will also scale.

iii) The shrinking of the outer arcs is parameterized by

$$
\begin{aligned}
& x_{1}^{2}=x_{c}^{2}+a \nu, \\
& x_{2}^{2}=x_{c}^{2}-a \mu, \quad \mu, \nu>0, \\
& p^{2}=x_{c}^{2}+a \pi .
\end{aligned}
$$


Expanding again in the complementary modulus $k^{2}$ leads to

$$
\frac{E(k)}{K(k)} \sim \mathcal{O}\left(\frac{1}{\ln a}\right)
$$

for the elliptic integrals and hence to the following behavior for the zero mode coefficients

$$
\begin{aligned}
\alpha_{1}, \alpha_{2} & \sim \mathcal{O}\left(\frac{1}{a \ln a}\right) \\
\alpha_{3} & =-\frac{x_{3}^{2}}{x_{c}^{2}-x_{3}^{2}}+\mathcal{O}\left(\frac{1}{\ln a}\right) .
\end{aligned}
$$

Although they become singular in the limit (6.15) they still remain subdominant, leading again to eq. (6.3) for $j=1,2$. The starting point for the iteration becomes like eqs. (6.10) and (6.11), with the index 3 replaced by 1 .

iv) The merging of the arcs while the inner arc is shrinking to zero is parameterized by

$$
\begin{aligned}
x_{2}^{2} & =a \nu, \\
x_{3}^{2} & =a \mu, \nu>\mu>0, \\
p^{2} & =a \pi .
\end{aligned}
$$

In this limit the modulus reaches

$$
k^{2}=1-\frac{\mu}{\nu}+\mathcal{O}(a)
$$

which is not necessarily small and may take any value in $(0,1)$. The $\alpha_{i}$ become

$$
\begin{aligned}
\alpha_{1} & =1+\mathcal{O}(a), \\
\alpha_{2} & =\frac{\nu}{\nu-\mu}\left(1-\frac{E(k)}{K(k)}\right)+\mathcal{O}(a), \\
\alpha_{3} & =\frac{1}{\nu-\mu}\left(-\mu+\nu \frac{E(k)}{K(k)}\right)+\mathcal{O}(a) .
\end{aligned}
$$

Consequently they do not contribute in the d.s.l..

v) In the case of merging arcs while the outer arcs are shrinking to zero the $\alpha_{i}$ will be sufficiently enhanced. The d.s.l. reads

$$
\begin{aligned}
x_{1}^{2} & =x_{c}^{2}+a \nu, \\
x_{2}^{2} & =x_{c}^{2}+a \mu, \nu>\mu>0, \\
x_{3}^{2} & =x_{c}^{2}, \\
p^{2} & =x_{c}^{2}+a \pi .
\end{aligned}
$$

In this limit the modulus becomes

$$
k^{2}=\frac{\mu}{\nu}+\mathcal{O}(a)
$$


which can reach any value in $(0,1)$. The zero mode coefficients now read

$$
\begin{aligned}
& \alpha_{1}=a^{-1} \frac{x_{c}^{2}}{(\nu-\mu)} \frac{E(k)}{K(k)}+\mathcal{O}\left(a^{0}\right), \\
& \alpha_{2}=a^{-1} \frac{x_{c}^{2}}{\mu}\left(1-\frac{\nu}{(\nu-\mu)} \frac{E(k)}{K(k)}\right)+\mathcal{O}\left(a^{0}\right), \\
& \alpha_{3}=a^{-1} \frac{x_{c}^{2}}{\mu}\left(-1+\frac{E(k)}{K(k)}\right)+\mathcal{O}\left(a^{0}\right) .
\end{aligned}
$$

In this case all $\alpha_{i}$ become sufficiently singular to contribute in the $\frac{d x_{i}^{2}}{d V}(p)$ in the d.s.l.. The iterative procedure will therefore not simplify and in addition the elliptic integrals remain explicitly present in the continuum limit. This interesting new continuum behavior has also been found in the hermitian model [8].

\section{The string susceptibility}

In this section a closed and universal expression will be given for the string susceptibility of both the complex and hermitian matrix model with two cuts. It reflects once more the fact that the two-point correlators of both models are universal as well. As the calculation for the complex model is much simpler it is presented in some detail. For the hermitian model only the final result will be given, which can be obtained along the same lines when starting from [8]. Both susceptibilities are shown to belong to the same class of universality. The knowledge of the string susceptibility may either serve to study the critical behavior of the model, as for example in 21] for the $O(n)$-model or in [22] for the Penner model. On the other hand when one tries to relate matrix models to integrable hierarchies using loop equation techniques [9, 10] the susceptibility enters directly the corresponding Gelfand-Dikii ansatz.

In order to introduce an overall coupling constant in front of the potential the following replacement is performed,

$$
V(p) \rightarrow \frac{1}{T} \tilde{V}(p)
$$

The parameter $T$ can be thought of as a temperature or the cosmological constant. The string susceptibility $U(T)$ is then obtained from the planar free energy $F_{0}$,

$$
\begin{aligned}
U(T) & =\frac{d^{2}}{d T^{2}}\left(T^{2} F_{0}\right) \\
& \sim\left(T_{c}-T\right)^{-\gamma_{s t r}} .
\end{aligned}
$$

Here it is supposed, that $U(T)$ shows some critical behavior at $T=T_{c}$. In order to exploit the knowledge of the quantities calculated so far as $W_{0}(p)$, the two following identities will be used, which can be shown easily from the definitions,

$$
\begin{aligned}
T^{2} \frac{d F_{0}}{d T} & =2 \oint_{\mathcal{C}} \frac{d \omega}{4 \pi i} W_{0}(\omega) \tilde{V}(\omega) \\
T \frac{d U}{d T} & =\frac{d^{2}}{d T^{2}}\left(T^{3} \frac{d F_{0}}{d T}\right)=\frac{d^{2}}{d T^{2}}\left(2 \oint_{\mathcal{C}} \frac{d \omega}{4 \pi i} T W_{0}(\omega) \tilde{V}(\omega)\right) .
\end{aligned}
$$


The final result obtained will thus be $\frac{d U}{d T}$. Taking $W_{0}(p)$ from eq. (3.4) together with the replacement (7.1) the first differentiation of the integrand yields

$$
\frac{d}{d T}\left(T W_{0}(\omega)\right)=\frac{1}{4} \sum_{i=1}^{s} T I_{i}^{(1)} \frac{d x_{i}^{2}}{d T} \frac{1}{\left(\omega^{2}-x_{i}^{2}\right) \phi^{(0)}(\omega)} .
$$

In order to proceed the quantities $\frac{d x_{i}^{2}}{d T}$ have to be determined, which can be done exactly along the same lines as the calculation of the $\frac{d x_{i}^{2}}{d V}(p)$. Here $s=3$ has to be specified to obtain explicit results. Differentiating the boundary equations (3.8) and (3.9) with respect to $T$ leads to the following set of equations

$$
\begin{aligned}
0 & =\sum_{i=1}^{3} T I_{i}^{(1)} \frac{d x_{i}^{2}}{d T}, \\
4 & =\sum_{i=1}^{3} x_{i}^{2} T I_{i}^{(1)} \frac{d x_{i}^{2}}{d T}, \\
0 & =\sum_{i=1}^{3} T I_{i}^{(1)} \frac{d x_{i}^{2}}{d T} K_{i} .
\end{aligned}
$$

The solution is obtained with the help of appendix B eq. (B.2), where the $K_{i}$ are given,

$$
T I_{i}^{(1)} \frac{d x_{i}^{2}}{d T}=\frac{4}{\prod_{j \neq i}\left(x_{i}^{2}-x_{j}^{2}\right)}\left(x_{i}^{2}-x_{3}^{2} \frac{\Pi(\alpha, k)}{K(k)}\right), \quad i=1,2,3 .
$$

Inserting this into eq. (7.4) for $s=3$ one arrives after some calculation at

$$
\frac{d}{d T}\left(T W_{0}(\omega)\right)=\phi^{(0)}(\omega)\left(\omega^{2}-x_{3}^{2} \frac{\Pi(\alpha, k)}{K(k)}\right),
$$

and consequently

$$
T \frac{d U}{d T}=\frac{d}{d T}\left(2 \oint_{\mathcal{C}} \frac{d \omega}{4 \pi i} \phi^{(0)}(\omega)\left(\omega^{2}-x_{3}^{2} \frac{\Pi(\alpha, k)}{K(k)}\right) \tilde{V}(\omega)\right) .
$$

As the differentiation of the complete elliptic integrals with respect to their moduli is known (e.g. in [20]), it is useful to rewrite $\frac{d}{d T}$ in the following way

$$
\frac{d}{d T}=\frac{\partial}{\partial T}+\sum_{i=1}^{s} \frac{d x_{i}^{2}}{d T} \frac{\partial}{\partial x_{i}^{2}}+\frac{d k^{2}}{d T} \frac{\partial}{\partial k^{2}}+\frac{d \alpha^{2}}{d T} \frac{\partial}{\partial \alpha^{2}} .
$$

Using this form on the r.h.s. of eq. (7.8) one arrives again after some tedious calculation at the following result for the integrand

$$
\begin{aligned}
& \frac{d}{d T}\left(\phi^{(0)}(\omega)\left(\omega^{2}-x_{3}^{2} \frac{\Pi(\alpha, k)}{K(k)}\right)\right)= \\
& =\frac{1}{2} \sum_{i=1}^{3} \frac{d x_{i}^{2}}{d T}\left(1-\frac{x_{3}^{2}}{x_{i}^{2}} \frac{\Pi(\alpha, k)}{K(k)}\right)\left(x_{i}^{2} \phi_{i}^{(1)}(\omega)+\alpha_{i} \phi^{(0)}(\omega)\right) .
\end{aligned}
$$


In order to get a universal expression for $\frac{d U}{d T}$ in eq. (7.8) one would like to perform a partial integration to arrive at an integral containing $V^{\prime}(\omega)$. Next the boundary conditions eqs. (3.8) and (3.9) would allow to eliminate the explicit dependence on the potential. Indeed the wanted transformation can be done due to the following observation. The last parenthesis in eq. (7.10) containing the $\omega$-dependence is precisely given by $I_{i}^{(1)} \frac{d x_{i}^{2}}{d V}(\omega)$, which can be written as a total derivative with respect to $\omega$. This becomes evident when rewriting the linear set of equations (B.1), which determine these quantities?,

$$
\begin{aligned}
0= & \sum_{i=1}^{3} I_{i}^{(1)} \frac{d x_{i}^{2}}{d V}(p)+\frac{\partial}{\partial p}\left(p \phi^{(0)}(p)\right), \\
0= & \sum_{i=1}^{3} x_{i}^{2} I_{i}^{(1)} \frac{d x_{i}^{2}}{d V}(p)+\frac{\partial}{\partial p}\left(p^{3} \phi^{(0)}(p)\right), \\
0= & \sum_{i=1}^{3} I_{i}^{(1)} \frac{d x_{i}^{2}}{d V}(p) K_{i}-\frac{\partial}{\partial p}\left(p \phi^{(0)}(p) A(p)\right), \\
& A(p)=\int_{x_{3}}^{x_{2}} d y \frac{1}{p^{2}-y^{2}} \frac{1}{\phi^{(0)}(y)}
\end{aligned}
$$

The last equation can be shown to hold using eq. (A.6). In the same way the boundary condition (3.9) is shown to be equivalent to

$$
0=\oint_{\mathcal{C}} \frac{d \omega}{4 \pi i} V^{\prime}(\omega) 2 \omega \phi^{(0)}(\omega) A(\omega) .
$$

From eq. (7.11) one obtains

$I_{1}^{(1)} \frac{d x_{1}^{2}}{d V}(p)=\frac{1}{\Sigma} \frac{\partial}{\partial p}\left(\left(K_{2}-K_{3}\right) p^{3} \phi^{(0)}(p)+\left(x_{2}^{2}-x_{3}^{2}\right) p \phi^{(0)}(p)+\left(x_{2}^{2}-x_{3}^{2}\right) p \phi^{(0)}(p) A(p)\right)$

where

$$
\Sigma=\left(x_{2}^{2}-x_{3}^{2}\right) K_{1}+\left(x_{3}^{2}-x_{1}^{2}\right) K_{2}+\left(x_{1}^{2}-x_{2}^{2}\right) K_{3} .
$$

The $\frac{d x_{i}^{2}}{d V}(p)$ for $i=2,3$ are obtained by a cyclic permutation of the indices. When inserting eq. (7.13) into eq. (7.10), integrating by parts in eq. (7.8) and using the boundary conditions $(3.8)$ and $(7.12)$ only the terms $\sim p^{3} \phi^{(0)}(p)$ will survive in eq. (7.13). The result for the string susceptibility finally reads

$$
\frac{d U}{d T}=\sum_{i=1}^{3} \frac{d x_{i}^{2}}{d T} \frac{2}{\prod_{j \neq i}\left(x_{i}^{2}-x_{j}^{2}\right)}\left(x_{i}^{2}-x_{3}^{2} \frac{\Pi(\alpha, k)}{K(k)}\right)^{2} .
$$

It is a remarkable universal quantity, as it shows no explicit potential dependence. Compared to the one-arc susceptibility

$$
\begin{aligned}
\frac{d U_{s=1}}{d T} & =\frac{2}{x^{2}} \frac{d x^{2}}{d T}, \\
\frac{d x^{2}}{d T} & =\frac{4}{T I^{(1)}},
\end{aligned}
$$

\footnotetext{
${ }^{5}$ The same can be achieved for the general set of equations (A.5) and A.6.
} 
which can be obtained as a simple exercise, eq. (7.15) contains much more structure and thus offers more possibilities for showing critical behavior.

The string susceptibility for the hermitian matrix model with two cuts can be calculated exactly along the same lines presented in this section, taking [8] as a starting point. It is given by

$$
\frac{d U^{H}}{d T}=\sum_{i=1}^{4} \frac{d \bar{x}_{i}}{d T} \frac{2}{\prod_{j \neq i}\left(\bar{x}_{i}-\bar{x}_{j}\right)}\left(\bar{x}_{i}-\bar{x}_{4}+\left(\bar{x}_{4}-\bar{x}_{3}\right) \frac{\Pi(\bar{\alpha}, \bar{k})}{K(\bar{k})}\right)^{2}
$$

where

$$
T \bar{I}_{i}^{(1)} \frac{d \bar{x}_{i}}{d T}=\frac{4}{\prod_{j \neq i}\left(\bar{x}_{i}-\bar{x}_{j}\right)}\left(\bar{x}_{i}-\bar{x}_{4}+\left(\bar{x}_{4}-\bar{x}_{3}\right) \frac{\Pi(\bar{\alpha}, \bar{k})}{K(\bar{k})}\right), i=1, \ldots, 4 .
$$

To compare with the result for the complex model eq. (7.15) $\bar{x}_{4}$ is set to zero, as being done already in section 1 . It can be seen now explicitly, that both string susceptibilities belong to the same universality class. This is not so astonishing, since the planar two-loop correlators of the complex and hermitian two-cut model have been identified already in section 5. For completeness the one-arc susceptibility is also given for the hermitian model,

$$
\begin{aligned}
\frac{d U_{s=1}^{H}}{d T} & =\sum_{i=1,2} \frac{d \bar{x}_{i}}{d T} \frac{2}{\prod_{j \neq i}\left(\bar{x}_{i}-\bar{x}_{j}\right)}, \\
T \bar{I}_{i}^{(1)} \frac{d \bar{x}_{i}}{d T} & =\frac{4}{\prod_{j \neq i}\left(\bar{x}_{i}-\bar{x}_{j}\right)}, \quad i=1,2 .
\end{aligned}
$$

\section{Conclusions}

In this paper the program of classifying and calculating correlation functions of the unitary ensembles, the hermitian and complex one-matrix model, is completed. For any number of arcs of the support of the eigenvalue density the correlation functions are found to be universal for both models, thus providing a complete classification of all possible correlators to occur. The higher order corrections in $1 / N^{2}$ to the correlators are determined by an iterative solution of the loop equation, where for genus one explicit results are obtained for any number of arcs. In general, in the double-scaling limit the one- and two-arc solution of the complex and hermitian model become equivalent all together. However, when the scaling limit is performed at merging or/and shrinking arcs, a new continuum behavior has been found here in the complex model as well as in the hermitian model [8].

An interesting question is to find applications for the multi-arc solutions, which were calculated here in the macroscopic large- $N$ limit. For example in solid states physics such situations with a Hamiltonian having a band structure for its eigenvalues typically occur.

It seems to be straightforward to analyze the critical behavior of the twoarc solutions now directly from the given string susceptibilities. This could be done in a similar way as the investigation of all possible transitions in the 
double-scaling limit in section 6. Another point left for future work is the relation between the multi-arc matrix models and integrable hierarchies. The use of loop equation techniques [9, 10] requires the knowledge of the string susceptibility as it enters directly the corresponding Gelfand-Dikii ansatz. In [1] the $\mathrm{mKdV}$ and a subset of the nonlinear Schrödinger (NLS) hierarchy has been proposed for the symmetric and non-symmetric two-arc solution of the hermitian matrix model. However, in the latter case the ansatz made there for the orthogonal polynomials is very doubtful [12], so it remains to be checked whether the same results can be obtained from the loop equations.

Acknowledgments: This work was supported by European Community grant no. ERBFMBICT960997. I would like to thank J. Ambjørn, C. Kristjansen and Yu. Makeenko for helpful discussions. In addition I wish to thank Yu. Makeenko for sharing with me his unpublished manuscript [10].

\section{A Derivation of the basis}

It can be easily shown by induction, that the functions given in eq. (4.7) without the restriction to the $\frac{d}{d V}$-part form a possible set of basis functions, satisfying eq. (4.4) up to the addition of allowed zero modes of eq. (4.3):

$$
\begin{aligned}
& \tilde{\chi}_{i}^{(n)}(p) \equiv \frac{1}{I_{i}^{(1)}}\left(\phi_{i}^{(n)}(p)-\sum_{k=1}^{n-1} \tilde{\chi}_{i}^{(k)}(p) I_{i}^{(n-k+1)}\right), i=1, \ldots, 2 s \quad, \quad n \in \mathrm{N}_{+}, \\
& \tilde{\psi}^{(n)}(p) \equiv \frac{1}{M^{(0)}}\left(\Omega^{(n)}(p)-\sum_{k=1}^{n-1} \tilde{\psi}^{(k)}(p) M^{(n-k)}\right), n \in \mathrm{N}_{+} .
\end{aligned}
$$

In order to absorb the $p$-dependence of these functions into derivatives of the loop insertion operator, the $\frac{d}{d V}(p)$-part of $\phi_{i}^{(n)}(p)$ and $\Omega^{(n)}(p)$ has to be calculated. The basis functions are then fixed uniquely by subtracting the remaining part, which will turn out to consist only of zero modes. Therefore the $\frac{d}{d V}$ -

derivative of the basic variables $I_{i}^{(k)}, M^{(k)}$ and $x_{i}^{2}$ has to be determined. This can be achieved by rewriting the loop insertion operator as in eq. (4.10) and applying it to the definitions (4.5). Using the identity

$$
\frac{\partial}{\partial V}(p) V^{\prime}(\omega)=\frac{-2 \omega p}{\left(p^{2}-\omega^{2}\right)^{2}}
$$

the results read

$$
\begin{aligned}
\frac{d I_{i}^{(k)}}{d V}(p) & =\left(k+\frac{1}{2}\right)\left(I_{i}^{(k+1)} \frac{d x_{i}^{2}}{d V}(p)-x_{i}^{2} \phi_{i}^{(k+1)}(p)\right)-\left(k+\frac{s-1}{2}\right) \phi_{i}^{(k)}(p) \\
& +\frac{1}{2} \sum_{\substack{j=1 \\
j \neq i}}^{s} \sum_{l=1}^{k} \frac{1}{\left(x_{j}^{2}-x_{i}^{2}\right)^{k-l+1}}\left(x_{j}^{2} \phi_{i}^{(l)}(p)-I_{i}^{(l)} \frac{d x_{j}^{2}}{d V}(p)\right) \\
& +\frac{1}{2} \sum_{\substack{j=1 \\
j \neq i}}^{s} \frac{1}{\left(x_{j}^{2}-x_{i}^{2}\right)^{k}}\left(I_{j}^{(1)} \frac{d x_{j}^{2}}{d V}(p)-x_{j}^{2} \phi_{j}^{(1)}(p)\right), i=1, \ldots, 2 s \text { (A.3) }
\end{aligned}
$$




$$
\begin{aligned}
\frac{d M^{(k)}}{d V}(p)= & -\frac{2 k+1}{2} \Omega^{(k+1)}(p)-\frac{1}{2} \sum_{i=1}^{s} \frac{1}{x_{i}^{2 k+2}}\left(x_{i}^{2} \phi_{i}^{(1)}(p)-I_{i}^{(1)} \frac{d x_{i}^{2}}{d V}(p)\right) \\
& -\frac{1}{2} \sum_{i=1}^{s}\left(\frac{d x_{i}^{2}}{d V}(p) \sum_{l=0}^{k} \frac{M^{(l)}}{x_{i}^{2(k-l+1)}}-\sum_{l=1}^{k} \frac{1}{x_{i}^{2(k-l+1)}} \Omega^{(l)}(p)\right) .
\end{aligned}
$$

The $\frac{d x_{i}^{2}}{d V}(p)$ can be obtained from solving a linear set of equations derived from the boundary conditions. Applying $\frac{d}{d V}(p)$ in the form of eq. 4.10) to the first set of boundary equations (3.8) leads to

$0=\sum_{i=1}^{s}\left(x_{i}^{k-1} I_{i}^{(1)} \frac{d x_{i}^{2}}{d V}(p)-x_{i}^{2} p^{k-1} \phi_{i}^{(1)}(p)\right)+(k-s) p^{k-1} \phi^{(0)}(p), k=1,3, \ldots, s$

For the second set of boundary equations (3.9) one gets

$$
\begin{aligned}
0= & \frac{1}{2} \frac{\partial}{\partial p} \int_{x_{2 j+1}}^{x_{2 j}} d y \frac{2 p}{p^{2}-y^{2}} \frac{\phi^{(0)}(p)}{\phi^{(0)}(y)}-\sum_{i=1}^{s} I_{i}^{(1)} \frac{d x_{i}^{2}}{d V}(p) K_{i, j} \\
= & \sum_{i=1}^{s}\left(x_{i}^{2} \phi_{i}^{(1)}(p)-I_{i}^{(1)} \frac{d x_{i}^{2}}{d V}(p)\right) K_{i, j}, \quad j=1, \ldots, \frac{s-1}{2} \\
& K_{i, j} \equiv \int_{x_{2 j+1}}^{x_{2 j}} d y \frac{\sqrt{\prod_{k=1}^{s}\left(y^{2}-x_{k}^{2}\right)}}{\left(y^{2}-x_{i}^{2}\right)}
\end{aligned}
$$

Making the ansatz

$$
I_{i}^{(1)} \frac{d x_{i}^{2}}{d V}(p)=x_{i}^{2} \phi_{i}^{(1)}(p)+\sum_{l=0}^{\frac{s-3}{2}} \alpha_{i, l} p^{2 l} \phi^{(0)}(p), \quad i=1, \ldots, 2 s
$$

where the sum is over the allowed zero modes in eq. (4.3), one arrives at the following set of equations,

$$
\begin{aligned}
& 0=\sum_{i=1}^{s}\left(\sum_{l=0}^{\frac{s-3}{2}} \alpha_{i, l} x_{i}^{2 k} p^{2 l}-\sum_{l=0}^{k-1} x_{i}^{2(k-l)} p^{2 l}\right)+(2 k+1-s) p^{2 k}, k=0, \ldots, \frac{s-1}{2} \\
& 0=\sum_{i=1}^{s} \sum_{l=0}^{\frac{s-3}{2}} \alpha_{i, l} K_{i, j} p^{2 l}, \quad j=1, \ldots, \frac{s-1}{2} .
\end{aligned}
$$

Comparing powers of $p$ leads to precisely $s(s-1) / 2$ linear equations determining the $\alpha_{i, l}, i=1, \ldots, s, l=0, \ldots, \frac{s-3}{2}$, completely as functions of $x_{i}$ and $K_{i, j}$, which are elliptic functions of the $x_{i}$. The $\alpha_{i, l}$ are thus uniquely determined by the support of the eigenvalue density $\sigma$. In appendix B they are calculated explicitly for the example $s=3$.

After having determined the $\frac{d x_{i}^{2}}{d V}(p)$ the $\frac{d}{d V}$-part of $\phi_{i}^{(k)}(p)$ and $\Omega^{(k)}(p)$ for the basis can now be obtained as follows. Resolving eqs. (A.3) and (A.4) for

\footnotetext{
${ }^{6}$ The argument $p$ has to be excluded to be between the cuts during the calculation and analytically continued in the end (compare to [8], appendix A).
} 
$\phi_{i}^{(k+1)}(p)$ and $\Omega^{(k+1)}(p)$, it becomes evident together with eq. A.7), that the part which is not expressed in terms of $\frac{d x_{i}^{2}}{d V}(p), \frac{d I_{i}^{(k)}}{d V}(p)$ or $\frac{d M^{(k)}}{d V}(p)$ is just a linear combination of allowed zero modes. These terms can now be subtracted in eq. (A.1) in order to achieve the unique basis eq. (4.7). The firsts terms needed for the results in eq. 4.8) read

$$
\begin{aligned}
\left.\phi_{i}^{(1)}(p)\right|_{\frac{d}{d V}-\text { part }}= & \frac{I_{i}^{(1)}}{x_{i}^{2}} \frac{d x_{i}^{2}}{d V}(p), \quad i=1, \ldots, s, \\
\left.\phi_{i}^{(2)}(p)\right|_{\frac{d}{d V}-\text { part }}= & \frac{I_{i}^{(2)}}{x_{i}^{2}} \frac{d x_{i}^{2}}{d V}(p)-\frac{2}{3 x_{i}^{2}} \frac{d I_{i}^{(1)}}{d V}(p)-\frac{2 I_{i}^{(1)}}{3 x_{i}^{4}} \frac{d x_{i}^{2}}{d V}(p) \\
& -\frac{I_{i}^{(1)}}{3 x_{i}^{2}} \sum_{\substack{j=1 \\
j \neq i}}^{s} \frac{1}{x_{j}^{2}-x_{i}^{2}}\left(\frac{d x_{j}^{2}}{d V}(p)-\frac{d x_{i}^{2}}{d V}(p)\right), \\
\left.\Omega^{(1)}(p)\right|_{\frac{d}{d V}-\text { part }}= & -2 \frac{d M^{(0)}}{d V}(p)+\sum_{i=1}^{s} \frac{1}{x_{i}^{2}} \frac{d x_{i}^{2}}{d V}(p) M^{(0)} .
\end{aligned}
$$

\section{B The $\frac{d x_{i}^{2}}{d V}(p)$ for $s=3$}

In this appendix the equations determining the zero mode coefficients $\alpha_{i, l}$ of the quantities $\frac{d x_{i}^{2}}{d V}(p)$ are explicitly solved for $s=3$. To start with the set of equations (A.5) and (A.6) for $s=3 \mathrm{read}$

$$
\begin{aligned}
& 0= \sum_{i=1}^{3}\left(I_{i}^{(1)} \frac{d x_{i}^{2}}{d V}(p)-x_{i}^{2} \phi_{i}^{(1)}(p)\right)-2 \phi^{(0)}(p), \\
& 0= \sum_{i=1}^{3} x_{i}^{2}\left(I_{i}^{(1)} \frac{d x_{i}^{2}}{d V}(p)-x_{i}^{2} \phi_{i}^{(1)}(p)\right)-\phi^{(0)}(p) \sum_{i=1}^{3} x_{i}^{2}, \\
& 0= \sum_{i=1}^{3}\left(I_{i}^{(1)} \frac{d x_{i}^{2}}{d V}(p)-x_{i}^{2} \phi_{i}^{(1)}(p)\right) K_{i}, \\
& K_{i} \equiv \int_{x_{3}}^{x_{2}} d y \frac{\sqrt{\prod_{k=1}^{3}\left(y^{2}-x_{k}^{2}\right)}}{y^{2}-x_{i}^{2}} .
\end{aligned}
$$

After substituting $y^{2} \rightarrow y$ in the elliptic integrals $K_{i}$ they can be found in [20], where they are expressed by the complete elliptic integrals of first, second and third kind:

$$
\begin{aligned}
K_{1} & =X\left(x_{2}^{2} x_{3}^{2} K(k)+x_{2}^{2}\left(x_{3}^{2}-x_{1}^{2}\right) E(k)+x_{3}^{2}\left(x_{1}^{2}-x_{2}^{2}-x_{3}^{2}\right) \Pi(\alpha, k)\right) \\
K_{2} & =X\left(\left(2 x_{1}^{2}-x_{2}^{2}\right) x_{3}^{2} K(k)+x_{2}^{2}\left(x_{3}^{2}-x_{1}^{2}\right) E(k)+x_{3}^{2}\left(-x_{1}^{2}+x_{2}^{2}-x_{3}^{2}\right) \Pi(\alpha, k)\right) \\
K_{3} & =X\left(\left(2 x_{1}^{2}-x_{3}^{2}\right) x_{2}^{2} K(k)+x_{2}^{2}\left(x_{3}^{2}-x_{1}^{2}\right) E(k)+x_{3}^{2}\left(-x_{1}^{2}-x_{2}^{2}+x_{3}^{2}\right) \Pi(\alpha, k)\right) \\
X & =\frac{1}{2} \frac{1}{\sqrt{x_{2}^{2}\left(x_{1}^{2}-x_{3}^{2}\right)}}, \quad \alpha^{2}=\frac{x_{2}^{2}-x_{3}^{2}}{x_{2}^{2}}, \quad k^{2}=\frac{x_{1}^{2}\left(x_{2}^{2}-x_{3}^{2}\right)}{x_{2}^{2}\left(x_{1}^{2}-x_{3}^{2}\right)} .
\end{aligned}
$$


The decomposition into $K(k), E(k)$ and $\Pi(\alpha, k)$ is not unique due to possible transformations of the moduli $k$ and $\alpha$. The ansatz (A.7) for $s=3$ reads

$$
I_{i}^{(1)} \frac{d x_{i}^{2}}{d V}(p)=x_{i}^{2} \phi_{i}^{(1)}(p)+\alpha_{i} \phi^{(0)}(p), \quad i=1,2,3,
$$

where $\alpha_{i} \equiv \alpha_{i, 0}$. Substituting it into eq. (B.1) one obtains the following linear set of equations for the $\alpha_{i}$

$$
\begin{aligned}
& 0=\sum_{i=1}^{3} \alpha_{i}-2 \\
& 0=\sum_{i=1}^{3}\left(\alpha_{i}-1\right) x_{i}^{2} \\
& 0=\sum_{i=1}^{3} \alpha_{i} K_{i} .
\end{aligned}
$$

With the help of eq. (B.2) the solution is obtained, which determines the $\frac{d x_{i}^{2}}{d V}(p)$ completely

$$
\begin{aligned}
\alpha_{1} & =1+\frac{x_{2}^{2}}{\left(x_{1}^{2}-x_{2}^{2}\right)} \frac{E(k)}{K(k)}, \\
\alpha_{1} & =\frac{x_{2}^{2}}{x_{2}^{2}-x_{3}^{2}}\left(1+\frac{\left(x_{3}^{2}-x_{1}^{2}\right)}{\left(x_{1}^{2}-x_{2}^{2}\right)} \frac{E(k)}{K(k)}\right), \\
\alpha_{1} & =\frac{x_{3}^{2}}{x_{3}^{2}-x_{2}^{2}}\left(1-\frac{x_{2}^{2}}{x_{3}^{2}} \frac{E(k)}{K(k)}\right) .
\end{aligned}
$$




\section{References}

[1] E. Brézin and A. Zee, Nucl. Phys. B402 (1993) 613

[2] E. Kanzieper and V. Freilikher, Phys. Rev. Lett.78 (1997) 3806

[3] E. V. Shuryak and J. J. M. Verbaarschot, Nucl. Phys. A560 (1993) 306

[4] J. Ambjørn, J. Jurkiewicz and Yu. Makeenko, Phys. Lett. B251 (1990) 517

[5] J. Ambjørn, C. F. Kristjansen and Yu. Makeenko, Mod. Phys. Lett. A7 (1992) 3187

[6] J. Ambjørn, L. Chekhov, C. F. Kristjansen and Yu. Makeenko, Nucl. Phys. B404 (1993) 127

[7] G. Akemann and J. Ambjørn, J. Phys. A29 (1996) L555

[8] G. Akemann, Nucl. Phys. B482 (1996) 403

[9] R. Dijkgraaf, H. Verlinde and E. Verlinde, Nucl. Phys. B348 (1991) 435

[10] Yu. Makeenko, An explicit solution of 2d topological gravity, unpublished, August 1991

[11] T. Hollowood, L. Miramontes, C. Nappi and A. Pasquinucci, Nucl. Phys. B373 (1992) 247

[12] R. C. Brower, N. Deo, S. Jain and C. Tan, Nucl. Phys. B405 (1993) 166

[13] Y. Morita, Y. Hatsugai and M. Kohmoto, Phys. Rev. B52 (1995) 4716

[14] C. W. J. Beenakker, Nucl. Phys. B422 (1994) 515

[15] C. Itoi, Nucl. Phys. B493 (1997) 651

[16] S. Higuchi, C. Itoi, S. Nishigaki and N. Sakai, Phys. Lett. B398 (1997) 123

[17] G. Akemann, Loop equations for multi-cut matrix models, ITP-UH-11-95, DESY 95-066, hep-th/9503185, March 1995

[18] S. Dalley, C. Johnson and T. Morris, Nucl. Phys. B368 (1992) 625

[19] J. Jurkiewicz, Phys. Lett. B245 (1990) 178;

F. David, Nucl. Phys. B348 (1991) 507

[20] P. F. Byrd and M. D. Friedman, Handbook of Elliptic Integrals for Engineers and Scientists, Springer Verlag, New York 1971

[21] B. Eynard and C. F. Kristjansen, Nucl. Phys. B455 (1995) 577; Nucl. Phys. B466 (1996) 463

[22] J. Ambjørn, C. F. Kristjansen and Yu. Makeenko, Phys. Rev. D50 (1994) 5193 